\title{
Effect of dietary fat on fat absorption and concomitant plasma and tissue fat composition in a rat model of short bowel syndrome
}

Published online: 25 March 2004

(C) Springer-Verlag 2004

\begin{abstract}
The aim of this study was to investigate the effect of dietary fat on the time course of changes in fat absorption and tissue and plasma lipid composition in a rat model of short bowel syndrome (SBS). Male Sprague-Dawley rats underwent either a bowel transection with re-anastomosis (Sham rats) or $75 \%$ small bowel resection (SBS rats). Animals were randomly assigned to one of three groups: Sham rats fed normal chow (ShamNC), SBS rats fed normal chow (SBS-NC), or SBS rats fed a high-fat diet (SBS-HFD). Rats were sacrificed on day 3 or 14. Body weight, food intake, food clearance (dry fecal mass), and fat clearance (total fecal fat) were measured twice a week. Fat and energy intakes were calculated according to the amount of ingested food. Food and fat absorbability were calculated as intake minus clearance and were expressed as percent of intake. Serum cholesterol, triglyceride, and albumin were measured. Total lipid composition of the liver, epididymal adipose tissue, and the small intestine was determined. Statistical analysis was performed by a Student's test, with $p$ values $<0.05$ considered significant. Both food and fat absorbability diminished after bowel resection in rats fed NC. This was accompanied by a decrease in body weight gain, plasma triglyceride and protein levels, and total lipid content of the liver at day 3 and of a decrease in adipose tissue at day 14 following operation. SBS-HFD rats experienced a significant increase $(p$ $<0.05$ ) in food absorbability after 7 days and fat absorbability after 3 days compared with Sham-NC and SBS-NC rats $(p<0.05)$, as well as increases in serum
\end{abstract}

I. Sukhotnik ( $\square)$

Carmel Medical Center, Dept. of Surg. B, 7 Michal str, 34362 Haifa, Israel

E-mail: igor-dr@internet-zahav.net

Tel.: + 972-4-8256815

Fax: +972-4-8346083

I. Sukhotnik · N. Mor-Vaknin · R. A. Drongowski A. G. Coran · C. M. Harmon

Section of Pediatric Surgery, C.S. Mott Children's Hospital and University of Michigan Medical School,

Ann Arbor, MI, USA cholesterol, triglycerides, and glucose compared with SBS-NC rats. On day 14, plasma lipid levels in SBSHFD rats were not different from SBS-NC or control rats; however, albumin levels were higher. A high-fat diet increased total fat content of the liver early after operation. In conclusion, in a rat model of SBS, an early high-fat diet increased the absorptive capacity of the intestinal remnant as seen by increased food and fat absorbability. These findings suggest a benefit of a highfat diet on intestinal adaptation in general and on lipid absorption in particular.

Keywords Short bowel syndrome - Dietary lipid · Fat absorption · Tissue lipids · Plasma lipids

\section{Introduction}

Short bowel syndrome (SBS) is a condition characterized by a loss of intestinal length or competence, resulting in a diminished ability to digest and absorb a regular diet [1]. Many authors have stated that a loss of $50 \%$ or more of small bowel length produces SBS $[2,3]$. Although the ability to absorb many nutrients is decreased following resection, lipid absorption is generally considered the most vulnerable $[4,5]$. The combined loss of absorptive surface area and compromised enterohepatic circulation [6], decreased bile acid pool [7, 8] and decreased pancreatic lipase secretion $[9,10]$ results in steatorrhea and inefficient fat absorption. Therefore, patients with SBS have been considered to benefit from a low-fat diet early in the course of therapy [2].

Enteral feeding is one of the major trophic factors in stimulating intestinal adaptation after small bowel resection [11]. However, not all nutrients have equal stimulating trophic effects. Among the factors promoting post-resection intestinal adaptation, long-chain fatty acids are considered the most effective [12]. It has been reported that animals with SBS receiving a diet deficient in essential fatty acids have significantly impaired 
intestinal mucosal hyperplasia [13]. Over the past decade, significant research has focused on the influence of long-chain fatty acids on intestinal adaptation in general $[4,12,13,14]$. Relatively little attention has been given to the effects of dietary lipids on lipid absorption in the very early postoperative period after bowel resection. In the present study, we investigated the effects of dietary fat on the time course of fat clearance, fat absorption, and concomitant changes in plasma and tissue total lipid composition in rats following massive bowel resection.

\section{Materials and methods}

Male Sprague-Dawley rats weighing 250-320 g were maintained in a controlled environment $\left(22^{\circ} \mathrm{C}\right.$ with a 12 -h light/dark cycle) and were given free access to water and standard rat chow (Research Diets, New Brunswick, NJ, USA). After an overnight fast, animals were anesthetized with sodium pentobarbital $(45 \mathrm{mg} / \mathrm{kg})$ administered intraperitoneally. Under sterile conditions, the abdomen was opened by midline incision. For SBS, a $75 \%$ resection was performed, preserving the vascular arcade and leaving $5 \mathrm{~cm}$ of proximal jejunum and $10 \mathrm{~cm}$ of distal ileum. Intestinal continuity was restored by an end-to-end single layer anastomosis using interrupted $6 / 0$ silk sutures (Ethicon, Somerville, NJ, USA). For Sham animals, the intestine was divided and re-anastomosed without resection. For all operations, the abdominal cavity was closed in two layers with running suture of 3/0 Dexon (Davis \& Geck, NY, USA). Antimicrobial cream with pramoxine $\mathrm{HCl}$ (Mycitracin Plus, Johnson \& Johnson, Skillman, NJ, USA) was applied to the incision for topical pain relief.

Following laparotomy, all animals had access to water for the first $24 \mathrm{~h}$ following operation. Rats were then given normal $(10 \%$ kcal fat) or high-fat ( $50 \% \mathrm{kcal}$ fat) diets and water ad lib for 3 or 14 days until sacrifice (Table 1) The rats were randomly assigned to one of three groups: (1) Sham-operated control rats fed normal chow (Sham-NC), (2) rats with SBS fed normal chow (SBS-NC), or (3) rats with SBS fed a high-fat diet (SBS-HFD).

Following operation, body weight and unconsumed food were weighed twice weekly. Food intake was calculated and expressed as $\mathrm{g}$ /day. Feces from each animal was collected during the 14 days of the experimental period, weighed, and frozen at $-18^{\circ} \mathrm{C}$ until analysis. To determine total fecal lipid, a sample of wet feces was emulsified and acidified with hydrochloric acid $(\mathrm{pH}<3), 5 \mathrm{ml}$

Table 1 Diet composition

\begin{tabular}{lll}
\hline $\begin{array}{l}\text { Ingredients } \\
(\mathrm{gm} \%)\end{array}$ & $\begin{array}{l}\text { Normal chow } \\
\text { (No 99031901) }\end{array}$ & $\begin{array}{l}\text { High-fat diet } \\
\text { (No D12450B) }\end{array}$ \\
\hline Casein, 80 mesh & 200 & 200 \\
L-cysteine & 3 & 3 \\
Cornstarch & 315 & 22.5 \\
Maltodextrin 10 & 35 & 125 \\
Sucrose & 350 & 147.5 \\
Cellulose, BW200 & 50 & 50 \\
Soybean oil & 25 & 25 \\
Lard & 20 & 200 \\
Minerals & 45 & 45 \\
Vitamins & 12 & 12 \\
Total: & & \\
Fat gm $\% / \mathrm{kcal} \%$ & $4.3 / 10$ & $27.1 / 49.9$ \\
Energy kcal/gm & 3.85 & 4.89 \\
\hline
\end{tabular}

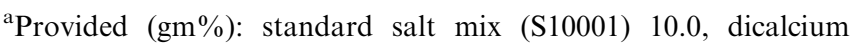
phosphate 13.0 , calcium carbonate 5.5 , potassium citrate 16.5 bupplied (mg\%): standard vitamin mix (V10001) 10.0, choline bitartrats 2.0 ethanol was added, and the mixture was extracted with $20 \mathrm{ml}$ of petroleum ether. Two additional extractions with $20-\mathrm{ml}$ portions of petroleum ether were performed. The petroleum ether was evaporated in a steam bath, the lipid extract was dried in a vacuum desiccator overnight, and the residue was weighed [15].

Energy and fat intake were calculated according to the amount of ingested food. The difference between the amount of food consumed and the amount of the dry feces was used as an estimate of absorbability [12]. The difference between the total $\mathrm{mg}$ of fat ingested and the total $\mathrm{mg}$ of fat remaining in the dry feces represented fat absorbability as previously described by other investigators $[5$, $16,17]$.

The rats were anesthetized with intraperitoneal sodium phenobarbital $(45 \mathrm{mg} / \mathrm{kg})$ and sacrificed by open pneumothorax on day 4 or 15 of the experiment. Blood samples for cholesterol, triglycerides, albumin, and glucose were obtained through immediate direct cardiac puncture. The blood was transferred to heparinized tubes, and plasma samples were obtained by centrifugation and stored at $-80^{\circ} \mathrm{C}$. The plasma concentrations of cholesterol, triglycerides, glucose, and total protein were measured spectrophotometrically using commercially available kits (Sigma, Kit \# 352, 339 , 16-UV and Bio-Rad, Protein Assay).

The heart, liver, kidneys, adrenal glands, lungs, brain, spleen, and testes were quickly removed, rinsed with water to remove remaining blood, blotted dry, and weighed. Total tissue lipid was measured in the liver, ileum, and white adipose tissue of the epididymis by the method of Bligh and Dyer [18]. Briefly, after homogenization, total lipids were extracted with chloroformmethanol $(1: 2, \mathrm{v} / \mathrm{v})$. The aqueous phase was aspirated and discarded, and the neutral lipids (chloroform phase) were transferred and then dried under nitrogen and weighed.

Statistical analysis

To assess the effect of bowel resection, SBS-NC rats were compared with Sham-NC rats. To determine the effect of a high-fat diet, SBSHFD rats were compared with SBS-NC rats. Animal and tissue weights were expressed as mean value \pm SEM. One-way ANOVA coupled with Duncan's multiple range tests were used to identify significant differences due to diet type ( $p$ values $<0.05$ were considered significant). When two groups of data were being compared, a Student's test was used.

\section{Results}

Body and organs weight

The animal weights at the onset of feeding were similar in all treatment groups $(240-250 \mathrm{~g})$. As expected, the Sham-operated control rats gained weight immediately after the operation, whereas the SBS animals lost weight after intestinal resection and then remained constant for the first 3 days. After the 3 rd postoperative day, the two groups of SBS rats gained weight similarly. There was a significant difference in the final weights between SBS and Sham rats, but no significant difference between SBS rats fed the two diets (Fig. 1).

At sacrifice, the weights ( $\mathrm{mg} / \mathrm{g}$ body weight) of the heart, lungs, liver, kidneys, spleen, and brain did not vary significantly among the groups (Table 2). However, the mean weight of the adrenals and testes significantly increased in the first 3 days in SBS rats compared with Sham-NC control animals. Following exposure to a high-fat diet for 14 days, SBS rats demonstrated an 


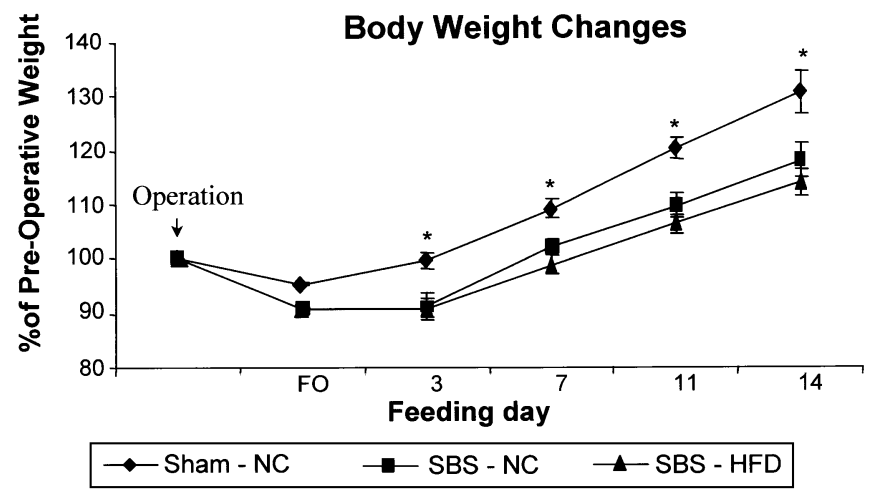

Fig. 1 Average weight changes in Sham-NC and SBS rats fed normal chow (NC) or a high-fat diet (HFD) for 2 weeks. Values are mean \pm SEM. Control $=$ preoperative weight, $\mathrm{FO}=$ feeding onset. $* p<0.05$ SBS rats vs. Sham-NC (control) rats

increase in brain and testes weight compared with SBS$\mathrm{NC}$ rats. Clinical signs of essential fatty acid deficiency (roughened or thin hair, scaly skin, hematuria, tail necrosis, growth reduction) [19] were not seen in the first 2 weeks after small bowel resection.

Energy intake, food, and fat balance

Energy intake decreased significantly in SBS rats compared with the control group (Fig. 2). In the SBS animals, exposure to a high-fat diet did not affect total energy intake.

Food intake was decreased in SBS-NC rats compared with Sham-operated control animals on days 3 and 14 (Fig. 3). However, food clearance (dry fecal mass) remained unchanged. By days 3 and 14, food absorbability decreased following massive bowel resection compared with Sham-NC rats, suggesting generalized malabsorption. Although both food intake and clearance decreased following exposure to a high-fat diet, the decrease of food clearance was more significant. Food absorbability in the SBS-HFD rats, therefore, was significantly greater on days 3 and 14 compared with the rats fed normal chow, suggesting that a high-fat diet improves generalized malabsorption in this model.

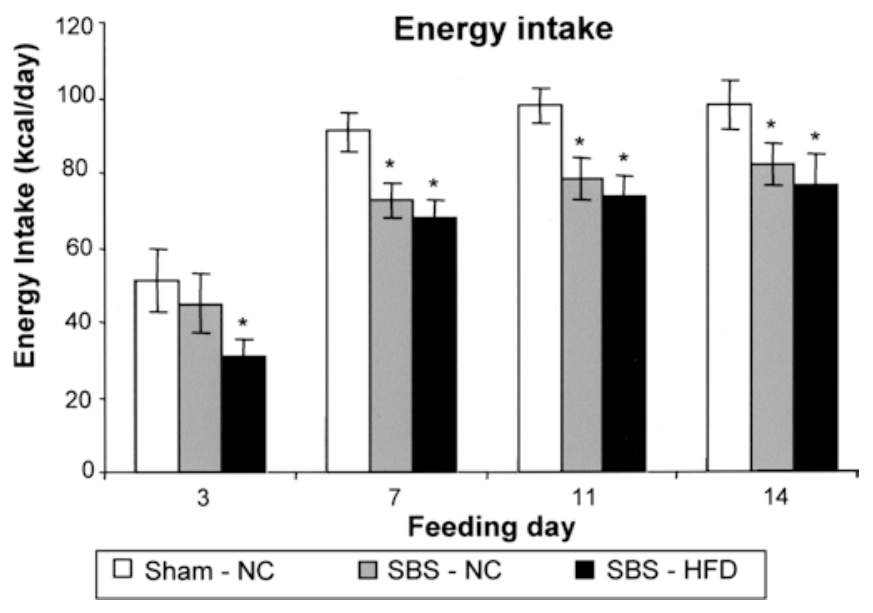

Fig. 2 Average energy intake in Sham-NC and SBS rats fed normal chow (NC) or a high-fat diet (HFD) for 2 weeks. Values are mean \pm SEM. $* p<0.05$ SBS vs. Sham-NC (control) rats. The difference between SBS-NC and SBS-HFD rats was not statistically significant

In accordance with food intake, a small but significant decrease in fat intake was seen in SBS-NC rats compared with the Sham-NC group (Fig. 4A). However, SBS-NC rats demonstrated a more marked increase in fat clearance on days 8-11 and 12-14 (Fig. 4B). Consequently, fat absorbability decreased following bowel resection in rats fed normal chow compared with their control counterparts, suggesting specific fat malabsorption (Fig. 4C). In contrast, rats fed the high-fat diet had significantly higher fat absorbability than both Sham-NC and SBS-NC rats throughout the study period, again suggesting that HFD improves lipid absorption.

Plasma lipids, glucose, and protein

Serum cholesterol, triglyceride, glucose, and protein levels at days 3 and 14 of dietary treatment are shown in Table 3. The SBS-animals fed normal chow had significantly lower plasma triglyceride and protein levels in the first 3 days than did Sham-NC rats. Also at day 3, SBS-HFD animals showed increased plasma cholesterol

Table 2 Tissue weight expressed on body weight basis in control (Sham) rats and rats with SBS fed $10 \mathrm{kcal} \%$ fat and $50 \mathrm{kcal} \%$ fat diets. Values are means \pm SEM. ( $N C$ normal chow, $H F D$ high-fat diet)

\begin{tabular}{|c|c|c|c|c|c|c|c|c|c|}
\hline $\begin{array}{l}\text { Experimental } \\
\text { groups }\end{array}$ & $\begin{array}{l}\text { Feeding } \\
\text { time }\end{array}$ & Heart & Liver & Lungs & Kidney & Adrenals & Spleen & Brain & Testes \\
\hline SBS-NC & 3 days & $3.0 \pm 0.1$ & $34.9 \pm 1.3$ & $4.6 \pm 0.1$ & $4.0 \pm 0.1$ & $0.25 \pm 0.01^{\mathrm{a}}$ & $2.7 \pm 0.3$ & $6.9 \pm 0.4$ & $6.1 \pm 0.1^{\mathrm{a}}$ \\
\hline SBS-HFD & 3 days & $3.0 \pm 0.1$ & $35.2 \pm 1.7$ & $4.5 \pm 0.1$ & $3.9 \pm 0.2$ & $0.29 \pm 0.02^{\mathrm{a}}$ & $2.3 \pm 0.2$ & $7.7 \pm 0.3$ & $6.2 \pm 0.2$ \\
\hline Sham-NC & 14 days & $2.7 \pm 0.1$ & $38.6 \pm 2.2$ & $4.5 \pm 0.2$ & $3.8 \pm 0.2$ & $0.17 \pm 0.01$ & $2.8 \pm 0.2$ & $5.6 \pm 0.3$ & $4.4 \pm 0.2$ \\
\hline SBS-NC & 14 days & $2.8 \pm 0.1$ & $40.1 \pm 0.8$ & $4.4 \pm 0.2$ & $3.9 \pm 0.2$ & $0.19 \pm 0.01$ & $2.8 \pm 0.1$ & $5.2 \pm 0.4$ & $4.5 \pm 0.3$ \\
\hline
\end{tabular}

\footnotetext{
${ }^{\mathrm{a}}$ Adrenals and testes weights in SBS-NC rats were significantly higher than in control rats $(p<0.05)$

${ }^{\mathrm{b}}$ Brain and testes weights were significantly higher in SBS-HFD rats than in SBS-NC rats $(p<0.05)$
} 


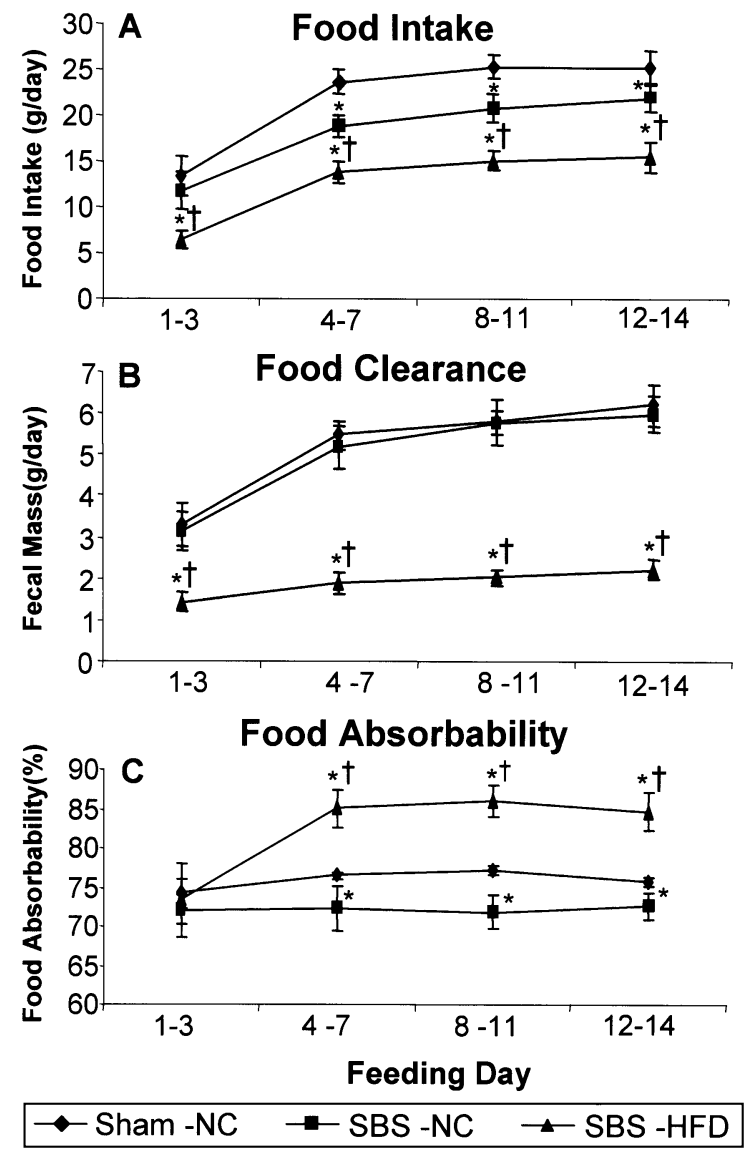

Fig. 3 Food input-output balance data expressed as net weight and as net absorption as a percent of intake. Values are means \pm SEM. Absorbability $=($ Intake - Clearance $) \times 100 /$ Intake. NC normal chow; SBS- short bowel syndrome rats; HFD- high-fat diet. ${ }^{*} p<$ 0.05 SBS vs. Sham-NC rats. $\dagger p<0.05$ SBS-HFD vs. SBS-NC rats

and increased triglyceride concentrations compared with Sham-NC and SBS-NC rats. SBS-HFD rats also had higher plasma protein levels compared with SBS-NC animals at day 3 . In contrast, by 14 days following operation, plasma lipid and glucose levels were unaffected by bowel resection or diet. However, plasma protein concentration remained decreased in SBS-NC rats compared with Sham-NC animals. Resected animals fed a high-fat diet for 14 days had significantly higher plasma protein levels than SBS-NC rats but had persistently lower protein levels compared with Sham controls.

Tissue lipids

The total lipid composition of the liver, ileal wall, and white adipose tissues of the epididymis is shown in Table 4. Compared with Sham-NC rats, SBS-NC rats had significantly lower total liver lipids at day 3 and significantly lower epididymal fat pad lipids on day 14 following operation. The SBS-HFD rats had a higher total liver lipid content than the SBS-NC rats on day 3.

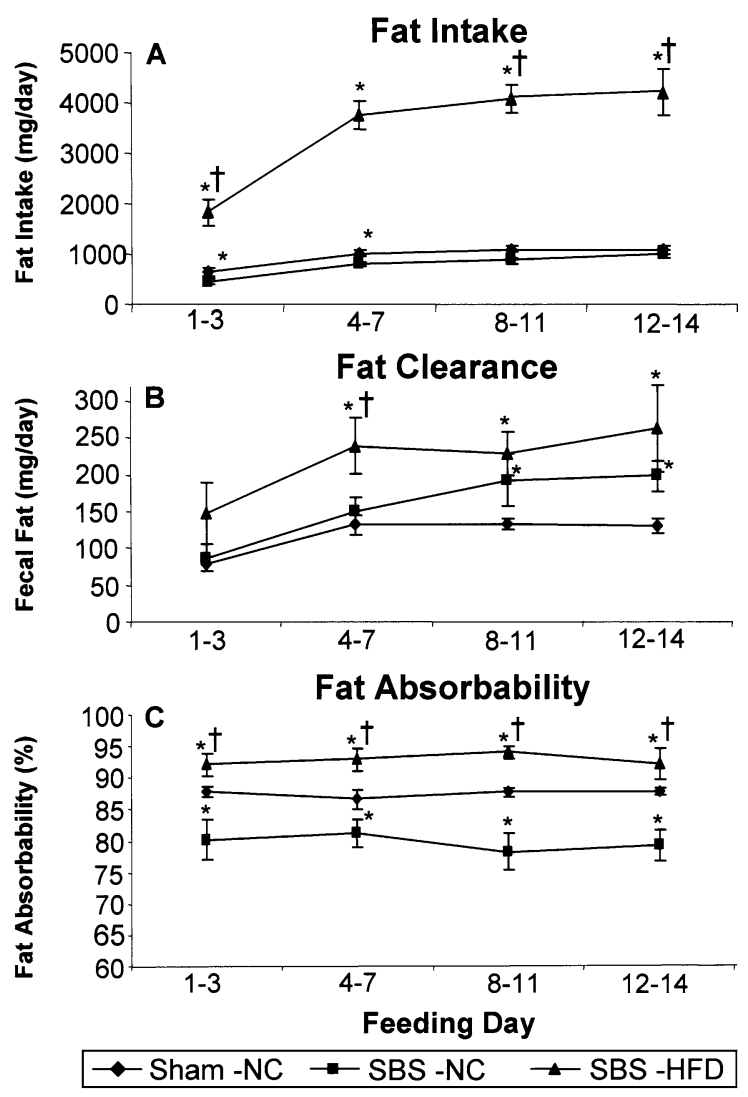

Fig. 4 Fat intake-clearance balance data. Values are means \pm SEM. Absorbability $=($ Intake - Clearance $) \times 100 /$ Intake. $\mathrm{NC}=$ normal chow; SBS = short bowel syndrome rats; HFD $=$ high-fat diet. $* p<0.05$ SBS vs. Sham-NC rats. $\dagger p<0.05$ SBS-HFD vs. SBS-NC rats

Tissue lipids from SBS-HFD rats were otherwise not statistically different from Sham-NC or SBS-NC rats on day 3. Exposure of SBS rats to a high-fat diet for 14 days resulted in a statistically significant increase in ileal wall lipid compared with Sham-NC rats, and a significant increase in epididymal white adipose lipids compared with SBS-NC rats.

\section{Discussion}

In SBS the bowel's ability to digest and absorb enteral nutrients is impaired, with lipid absorption generally considered the most compromised, leading to clinical problems such as malnutrition and steatorrhea [5, 20, 21]. The initial management of patients with SBS typically involves total parenteral alimentation, followed by gradual introduction of enteral feedings. It is well documented that exposure to intraluminal nutrients is required for stimulation of intestinal adaptation in general and for mucosal hyperplasia in particular [22, 23]. Therefore, many experts in the management of SBS recommend enteral feeding attempts as early as possible [16]. However, dietary recommendations in SBS are controversial, ambiguous, and subject to rapid varia- 
Table 3 Plasma lipids, glucose, and protein concentration in control and SBS rats fed normal chow $(N C)$ or a high-fat diet $(H F D)$. Values are means \pm SEM

\begin{tabular}{|c|c|c|c|c|c|}
\hline & Experimental groups & $\begin{array}{l}\text { Cholesterol } \\
(\mathrm{mg} / \mathrm{dl})\end{array}$ & $\begin{array}{l}\text { Triglyceride } \\
(\mathrm{mg} / \mathrm{dl})\end{array}$ & $\begin{array}{l}\text { Glucose } \\
(\mathrm{mg} / \mathrm{dl})\end{array}$ & $\begin{array}{l}\text { Protein } \\
(\mathrm{mg} / \mathrm{ml})\end{array}$ \\
\hline \multirow[t]{2}{*}{3 days } & Sham-NC & $55.3 \pm 8.2$ & $58.8 \pm 5.5$ & $128.1 \pm 16.9$ & $66.8 \pm 6.4$ \\
\hline & SBS-HFD & $82.9 \pm 5.7^{\mathrm{a}, \mathrm{b}}$ & $62.5 \pm 15.6$ & $171.0 \pm 18.2^{\mathrm{b}}$ & $51.9 \pm 4.3^{\mathrm{a}, \mathrm{b}}$ \\
\hline \multirow[t]{2}{*}{14 days } & Sham-NC & $60.5 \pm 3.3$ & $82.9 \pm 8.2$ & $136.6 \pm 9.8$ & $75.5 \pm 6.4$ \\
\hline & SBS-NC & $62.3 \pm 6.6$ & $74.0 \pm 4.2$ & $158.2 \pm 12.3$ & $31.4 \pm 6.6^{\mathrm{a}}$ \\
\hline
\end{tabular}

${ }^{\mathrm{a}} p<0.05$ SBS vs. Sham-NC rats

${ }^{\mathrm{b}} p<0.05$ SBS-HFD vs. SBS-NC rats

Table 4 Total lipid content of several tissues in normal rats and in rats with SBS fed normal chow (NC) or a high-fat diet (HFD). Values are means \pm SEM. (WAT epididymal white adipose tissue)

\begin{tabular}{lllll}
\hline & Experimental groups & $\begin{array}{l}\text { Liver lipid } \\
\text { (mg/g tissue) }\end{array}$ & $\begin{array}{l}\text { Intestinal lipid } \\
\text { (mg/g tissue) }\end{array}$ & $\begin{array}{l}\text { WAT lipid } \\
\text { (mg/g tissue) }\end{array}$ \\
\hline 3 days & Sham-NC & $40.1 \pm 2.3$ & $26.3 \pm 4.4$ & $22.3 \pm 6.0$ \\
& SBS-NC & $30.1 \pm 4.2^{\mathrm{a}}$ & $26.6 \pm 5.4$ & $24.9 \pm 4.4$ \\
14 days & SBS-HFD & $43.6 \pm 4.7^{\mathrm{b}}$ & $27.0 \pm 7.6$ & $17.9 \pm 2.0$ \\
& Sham-NC & $33.9 \pm 2.2$ & $25.2 \pm 3.0$ & $30.7 \pm 4.7$ \\
& SBS-NC & $38.3 \pm 1.9$ & $30.5 \pm 5.5^{\mathrm{a}}$ & $17.9 \pm 3.1^{\mathrm{a}}$ \\
\hline
\end{tabular}

${ }_{p}^{\mathrm{a}} p<0.05$ SBS vs. Sham-NC rats

${ }^{\mathrm{b}} p<0.05$ SBS-HFD vs. SBS-NC rats

tions. Patients with SBS are often given elemental diets containing medium-chain lipids during the early stages of enteral feeding. Fats contain the highest caloric density compared with other nutrients; however, long-chain saturated fats are considered the least well tolerated nutrient in SBS [1]. Absorption studies indicate that infants with SBS may have prolonged fat and bile acid malabsorption, with low serum levels of vitamin D and cholesterol but a normal absorptive capacity for other nutrients such as carbohydrates and amino acids [24]. Many authors have documented reduced fat absorption in older patients with SBS. Woolf et al. [25] studied eight patients with SBS who were clinically stable for at least 1 year and determined that fat absorption continued to be $54 \%$ of normal. Schwartz et al. described five patients who, after bowel resection, absorbed only about half of normal dietary fat intake [26]. As a consequence, widespread clinical practice is to avoid dietary fats in patients after massive small bowel resection.

Paradoxical to the observation that dietary fat is the least well tolerated nutrient early in SBS is the evidence that long-chain fats appear to be more effective stimulators of intestinal adaptation than either medium-chain fats or carbohydrates [12]. Therefore, several investigators have recommended adding long-chain fatty acids to diets of patients with SBS $[12,13,14]$. Although the role of long-chain fats in intestinal adaptation after bowel resection has been studied, relatively little attention has been given to in vivo metabolism of ingested fat in general. In addition, no study has investigated the effect of dietary fat intake on specific intestinal lipid absorption in the early postoperative period. Singh et al. studied adaptive changes in the small bowel in normal rats in response to increased lipid content of the diet and revealed that a high-fat diet increased uptake and reesterification of digested lipid by the small intestine [27].

In this work we studied the early effects of massive small bowel resection and a high-fat diet on intestinal lipid absorption using a rat model of SBS. In addition, in order to gain insight into the in vivo metabolic consequences of a high-fat diet in the setting of SBS, we evaluated several nutritional and metabolic parameters, including plasma cholesterol, triglycerides, glucose, and protein and tissue lipid content.

In our experimental model, massive bowel resection in rats fed normal chow produced a significant decrease in food absorbability, suggesting food malabsorption, and a decrease in fat absorbability, suggesting fat malabsorption compared with control animals. Decreased body weight gain and significantly decreased plasma triglycerides and protein levels accompanied the changes in food and fat absorption rates, signifying in vivo metabolic alterations secondary to SBS. In addition, SBS$\mathrm{NC}$ rats demonstrated lower liver total lipid levels at postoperative day 3 and lower adipose lipid at day 14, compared with the Sham-NC group. These findings suggest increased lipid metabolism (liver) and mobilization (adipose) to meet SBS nutritional demands as a consequence of decreased intestinal nutrient absorption. These observations comparing control Sham-NC animals with SBS-NC animals validate this model as an acceptable model of SBS for our particular study. 
In our study, early exposure of SBS animals to a high-fat diet led to a decrease in total food intake compared with SBS animals receiving a normal chow diet. More notable was the finding that total food absorbability was significantly increased in SBS-HFD rats compared with SBS rats fed NC as well as Sham$\mathrm{NC}$ control animals. As a result of increased absorbability and a higher caloric content in the high-fat diet, energy intake and weight gain were not significantly different between SBS-HFD animals and SBS rats fed normal chow. Interestingly, we also found that by day 14 , brain and testis weights increased significantly in SBS-HFD compared with SBS-NC and Sham-NC animals. In addition, both SBS-HFD and SBS-NC rats demonstrated increased adrenal and testis weights at the 3rd day following bowel resection compared with Sham$\mathrm{NC}$ controls. The implications of these observations are uncertain; however, changes in tissue mass have never been studied in a rat model of SBS. Roshanai and Sanders reported that a $40 \%$ kcal fat diet decreased the average liver mass in normal rats compared with rats fed normal chow [28]. Piche and Mahadevappa reported that the mean weight of the kidneys from rats fed a diet rich in highly unsaturated fatty acids was significantly higher than from those fed other diets [29]. Our results suggest that an early effect of bowel resection or SBS is increased adrenal and testis weights and that an effect of a high-fat diet in the setting of SBS is increased brain and testis weights.

Fat absorbability in SBS-HFD animals increased immediately after bowel resection and continued to increase during the time course of the experiment. These animals also demonstrated increased plasma and liver lipid content at day 3, increased adipose tissue lipid at day 14 , and increased plasma protein levels at both days 3 and 14 following operation. These results suggest that with exposure to increased dietary fat, the remaining bowel, after massive resection, responds in an effort to increase intestinal lipid absorptive capacity. Furthermore, increased lipid absorption in the setting of SBS appears to alter in vivo metabolic parameters in favor of early (day 3) lipid utilization and perhaps subsequent (day 14) lipid storage. Specific information about in vivo lipid metabolism in the setting of SBS (such as lipoprotein synthesis by the liver, fatty acid oxidation by muscle, or fatty acid esterification by adipocytes) is not provided by our study. However, our results set a foundation for further study on the effects of dietary fat on intestinal adaptation in SBS in general and on intestinal lipid absorption and physiologic in vivo lipid metabolism in particular.

In conclusion, in a rat model of SBS, an early high-fat diet appears to augment and accelerate functional parameters of intestinal adaptation. This is evident by increased intestinal total food and lipid absorption in SBS animals fed a high-fat diet compared with those fed normal chow. Increased nutrient absorption by the intestine is expected to have a positive metabolic impact on patients with SBS. These findings suggest that the early use of a high-fat diet in patients with SBS may enhance intestinal function and overall metabolic well being.

\section{References}

1. Taylor SF, Sokol RJ (1995) Infants with short bowel syndrome. In: Hay WW (ed) Neonatal nutrition and metabolism. Mosby, St. Louis, pp 432-450

2. Biller JA (1987) Short bowel syndrome. In: Grand RI, Sutphen JL, Dietz WH (eds) Pediatric nutrition. Theory and practice. Butterworth, Stoneham, MA, pp 481-487

3. Vanderhoof JA (1996) Short bowel syndrome. Neonatal Gastroenterol 23:377-386

4. Vanderhoof JA, Burkley KT, Antonson KT (1983) Potential for mucosal adaptation following massive small bowel resection in 3-week-old versus 8-week-old rats. J Pediatr Gastroenterol Nutr 2:672-676

5. Chiba T, Ohi R (1998 ) Do we still need to collect stool? Evaluation of visualized fatty acid absorption: experimental studies using rats. J Parenter Enteral Nutr 22:22-26

6. Molina MT, Ruiz-Cutierrez V, Vazquez CM (1990) Changes in uptake of linoleic acid and cholesterol by jejunal sacs of rats in vitro, after distal small bowel resection. Scand J Gastroenterol 25:613-621

7. Mok HYI, Parry PM, Dowling RH (1974) The control of bile acid pool size: effect of jejunal resection and phenobarbital on bile acid metabolism in the rats. Gut 15:247-253

8. Tilson MD, Boyer JL, Wright HK (1975) Jejunal absorption of bile salts after resection of the ileum. Surgery 77:231-234

9. Pitchumoni CS (1973) Pancreas in primary malnutrition disorders. Am J Clin Nutr 26:374-379

10. Weser E, Heller R, Tawil T(1977) Stimulation of mucosal growth in the rat ileum by bile and pancreatic secretion after jejunal resection. Gastroenterology 73:524-529

11. Menge H, Grafe M, Lorenz-Meyer H, Riecken EO (1975) The influence of food intake on the development of structural and functional adaptation following ileal resection in the rat. Gut 16:468-472.

12. Vanderhoof JA (1996) Short bowel syndrome. Neonatal Gastroenterol 23:377-386

13. Hart MH, Grandjean CJ, Park JHY, Erdman SH, Vanderhoof JA (1988) Essential fatty acid deficiency and postresection mucosal adaptation in the rats. Gastroenterology 94:682-687

14. Park JHY, Grandjean CJ, Hart MH, Vanderhoof JA (1989) Effects of dietary linoleic acid on mucosal adaptation after small bowel resection. Digestion 44:57-65

15. Henry RJ, Cannon DC, Winkelman JW (1974) Clinical chemistry: principles and technics, 2nd edn. Harper \& Row, New York, pp 1481-1483

16. Wilson MD, Blake WL, Salati LM (1990) Potency of polyunsaturated and saturated fats as short-term inhibitors of hepatic lipogenesis in rats. J Nutr 120:544-552

17. Wilson HD, Schedl HP (1987) Resection of rat small intestine: calcium, phosphorus, and fat balances and 1,25-dihydroxycholecalciferol. Am J Clin Nutr 45:437-442

18. Bligh EG, Dyer WJ (1959) A rapid method of total lipid extraction and purification. Can J Biochem Physiol 37:911-918

19. Holman RT (1968) Essential fatty acids deficiency. Prog Chem Fats Other Lipids 9: 275-348

20. Thomson AB (1986) Defined formula diets after jejunal and colonic uptake of lipids in rabbits with intact intestinal tract and following ileal resection. Res Exp Med 186:413-426

21. Booth IW, Lander AD (1998) Short bowel syndrome. Bailliere's Clinical Gastroenterology 12:739-772

22. Wesser E (1979) Nutritional aspects of malabsorption: short gut adaptation. Am J Med 67:1014-1019

23. Levine GM, Deren JJ, Yezdimir E (1976) Small-bowel resection: oral intake is the stimulus for hyperplasia. Digest Dis 21:542-546 
24. Ohkohchi N, Igarashi Y, Tazawa Y, Kobayashi Y, Ohi R, Kasai M (1986) Evaluation of the nutritional condition and absorptive capacity of nine infants with short bowel syndrome. J Ped Gastroenterol Nutr 5:198-206

25. Woolf GM, Miller C, Kurian R, Jeejeebhoy KN (1987) Nutritional absorption in short bowel syndrome: evaluation of fluid, calorie, and divalent cation requirements. Dig Dis Sci 32:8-15

26. Schwartz MK, Medwid A, Roberts KE, et al. (1955) Fat and nitrogen metabolism in patients with massive small bowel resections. Surg Forum 6:385-390
27. Singh A, Balint JA, Edmonds RH, Rodgers JB (1972) Adaptive changes of the small intestine in response to a high fat diet. Biochim Biophys Acta 260:708-715

28. Roshanai F, Sanders TAB (1985) Influence of different supplements of $n-3$ polyunsaturated fatty acids on blood and tissue lipids in rats receiving high intake of linoleic acid. Ann Nutr Metab 29:189-196

29. Piche LA, Mahadevappa VG (1990) Modification of rat platelet fatty acid composition by dietary lipids of animal and vegetable origin. J Nutr 120:444-449 